\section{Neonato masculino con síndrome Down, tetralogía de Fallot y enterocolitis necrosante}

\section{Male infant with Down syndrome, tetralogy of Fallot and necrotizing enterocolitis.}

Flores-Arizmendi $\mathrm{KA}^{1}$, Corcuera-Delgado $\mathrm{CT}^{2}$, Reyes-Macedo $\mathrm{M}^{3}$

\section{RESUMEN DE LA HISTORIA CLÍNICA}

Paciente masculino que ingresó a nuestro instituto a los dos días de nacimiento, referido de una clínica privada de la Ciudad de México con los diagnósticos de: recién nacido de término, con peso bajo para edad gestacional, restricción decrecimiento intrauterino y probable trisomía 21.

Contaba con los siguientes antecedentes de importancia: madre de 26 años, padre de 19 años de edad, dos medias hermanas, todos referidos sanos. Producto del tercer embarazo, diagnosticado desde el mes de gestación. Se refirió que la madre ingirió ácido fólico y sulfato ferroso, acudió a 8 consultas prenatales y se realizaron 3 ultrasonidos obstétricos que fueron reportados como normales. Nació en clínica privada a las 37.1 semanas de gestación por vía abdominal, por ruptura prematura de membranas de 12 horas de evolución y oligohidramnios. Las condiciones al nacimiento reportadas fueron Apgar 8/9, peso 1,820 kg, talla $43 \mathrm{~cm}$, perímetro cefálico $30 \mathrm{~cm}$, perímetro torácico $26 \mathrm{~cm}$, perímetro abdominal $25 \mathrm{~cm}$. Inició alimentación en hospital de origen, con fórmula para prematuro por sonda orogástrica.

A las 24 horas de nacimiento se solicitó biometría hemática, la cual reportó: hemoglobina $22.5 \mathrm{~g} / \mathrm{dL}$ hematócrito $59.7 \%$, leucocitos 27,750 células/mcL, neutrófilos 20,812 células/mcL,
${ }^{1}$ Médico adscrito a la Clínica de síndrome de Down. ${ }^{2}$ Médico adscrito al departamento de Anatomía Patológica.

${ }^{3}$ Médico residente del segundo año de patología pediátrica.

Instituto Nacional de Pediatría, México.

Recibido: 28 de marzo del 2017

Aceptado: 28 de marzo del 2017

Correspondencia

Dra. Karla Adney Flores-Arizmendi

adneyss@gmail.com

Este artículo debe citarse como

Flores-Arizmendi KA, Corcuera-Delgado CT, Reyes-Macedo M. Neonato masculino con síndrome Down, tetralogía de Fallot y enterocolitis necrosante. Acta Pediatr Mex. 2017;38(3):175-181. 
linfocitos 5,225 células/mcL, plaquetas 41,000/ $\mathrm{mcL}$, considerándolo potencialmente infectado, motivo por el cual se envió a esta institución.

A su ingreso en nuestra institución se detectó fontanela anterior amplia de $5 \times 3 \mathrm{~cm}$, normotensa, frente estrecha, perfil facial plano, puente nasal deprimido, nariz ancha, epicanto bilateral, fisuras palpebrales horizontalizadas, pabellones auriculares displásicos, aparente micrognatia, cuello corto con piel redundante, soplo sistólico expulsivo en foco pulmonar grado II/VI, sin hepatomegalia, abdomen blando, no se auscultó peristalsis, pulsos de adecuada intensidad en las cuatro extremidades. Peso $1,820 \mathrm{~kg}$, talla $43 \mathrm{~cm}$, frecuencia cardíaca 135 latidos por minuto, frecuencia respiratoria 28 respiraciones por minuto, temperatura $36.8^{\circ} \mathrm{C}$, tensión arterial 96/68 $\mathrm{mmHg}$.

Ingresó al servicio de neonatología y se inició alimentación con leche humana a libre demanda o fórmula de inicio al $13 \% 90 \mathrm{~mL} / \mathrm{kg} /$ día en 6 tomas y esquema antibiótico con ampicilina $100 \mathrm{mg} / \mathrm{kg} /$ día y amikacina $15 \mathrm{~mL} / \mathrm{kg} /$ día. A su ingreso se solicitaron estudios de laboratorio que reportaron: biometría hemática: hemoglobina $17.8 \mathrm{~g} / \mathrm{dL}$ hematocrito $55.6 \%$, leucocitos 9,200 células/mcL, neutrófilos totales 1,700 células/mcL, linfocitos totales 700 células/mcL, 74 mil plaquetas $/ \mathrm{mcL}$, proteína C reactiva $10.78 \mathrm{mg} /$ $\mathrm{dL}$, sodio $147 \mathrm{mEq} / \mathrm{L}$, potasio $5.2 \mathrm{mEq} / \mathrm{L}$, cloro $120 \mathrm{mEq} / \mathrm{L}$, calcio $7.4 \mathrm{mEq} / \mathrm{L}$, fósforo $6.8 \mathrm{mEq} / \mathrm{L}$ magnesio $1.9 \mathrm{mEq} / \mathrm{L}$, hormona estimulante de tiroides $1.92 \mathrm{mUI} / \mathrm{L}$, glucosa $59 \mathrm{mg} / \mathrm{dL}$, creatinina $1.24 \mathrm{mg} / \mathrm{dL}$, BUN $12.5 \mathrm{mg} / \mathrm{dL}$, urea $26.8 \mathrm{mg} / \mathrm{dL}$.

En su segundo día de estancia se valoró por el servicio de genética, quienes consideraron diagnóstico clínico de síndrome de Down y se citó para estudio citogenético.

Por su parte, el servicio de cardiología pediátrica realizó ecocardiograma que mostró situs solitus abdominal, tamaño de cavidades normales, concordancia veno-atrial, atrioventricular y ventrículo arterial. Se observó defecto septal auricular tipo ostium seccundum amplio, de $6 \times 7 \mathrm{~mm}$, con corto circuito de izquierda a derecha y gradiente de $3 \mathrm{mmHg}$, existía cabalgamiento del $50 \%$ de la aorta, con comunicación interventricular de entrada de $6 \mathrm{~mm}$, estenosis valvular pulmonar con gradiente de $18 \mathrm{mmHg}$, y gradiente infundibular de $7 \mathrm{mmHg}$. El anillo pulmonar con diámetro de $5 \mathrm{~mm}$ (puntaje Z -2.19), la rama derecha de la arteria pulmonar de $4 \mathrm{~mm}$ (puntaje $Z+0.08$ ) y se observó la rama izquierda de la arteria pulmonar con estenosis en su origen, diámetro de $2 \mathrm{~mm}$ (puntaje Z -3.77), gradiente aórtico de $2 \mathrm{mmHg}$. Insuficiencia tricuspídea leve con gradiente de $52 \mathrm{mmHg}$, lo que estimó una presión de salida de arteria pulmonar máxima de $62 \mathrm{mmHg}$. Cualitativamente se observó adecuada función ventricular, el arco aórtico derecho sin obstrucciones, se observó conducto arterioso permeable con boca aórtica de $4 \mathrm{~mm}$, pulmonar de $3 \mathrm{~mm}$ y diámetro de $5 \mathrm{~mm}$. Concluyendo diagnóstico: cardiopatía congénita de flujo pulmonar disminuido, tipo tetralogía de Fallot, asociado a comunicación inter-auricular tipo ostium seccundum y persistencia de conducto arterioso de $4 \times 3 \times 5 \mathrm{~mm}$.

El día tres de estancia hospitalaria hubo distensión abdominal, gasto por sonda orogástrica en pozos de café y evacuaciones con sangre macroscópica; se solicitó placa simple de abdomen donde se observó neumatosis intestinal. Se valoró por cirugía quienes colocaron catéter por venodisección en safena derecha, indicaron ayuno y cambio de manejo antibiótico a cefotaxime y metronidazol.

El servicio de hematología hizo una valoración y reportaron frotis de sangre periférica: serie roja con anisocitosis ++ esferocitos. Serie blanca con eosinófilos $1 \%$, juveniles $3 \%$, bandas $9 \%$, segmentados $16 \%$, linfocitos $17 \%$, monocitos $4 \%$ 
(al 50\%), 5 normoblastos. Serie plaquetaria: muy disminuida por apreciación: 1 cada 2-3 campos y algunas gigantes. Consideraron paciente con bicitopenia candidato a aspirado de médula ósea, al encontrarse estable para el procedimiento. Se indicó transfusión de plaquetas cada 8 horas y se inició vitamina $\mathrm{K}$.

El paciente presentó deterioro clínico con choque mixto, enterocolitis necrosante IIIA por la presencia de afectación sistémica grave: hipotensión arterial, signos evidentes de choque y deterioro ventilatorio. Requirió manejo con milrinona $0.5 \mathrm{mcg} / \mathrm{kg} / \mathrm{min}$, dopamina a $20 \mathrm{mcg} /$ $\mathrm{kg} / \mathrm{min}$ y dobutamina a $10 \mathrm{mcg} / \mathrm{kg} / \mathrm{min}$. Presentó anuria de 12 horas de evolución.

Se solicitaron radiografías seriadas de abdomen, en las que se observó persistencia de edema de pared abdominal, mala distribución de aire, ausencia de aire en zona distal, asa fija en doble riel en cuadrante superior derecho, sin observarse datos de perforación intestinal. Ante la gravedad del paciente, el servicio de cardiología hizo una revaloración y refirieron que a pesar de cursar con estenosis pulmonar tenía colateralaorto pulmonar grande con corto circuito de izquierda a derecha que generaba hiperflujo pulmonar, por lo que se suspendió milrrinona, ya que es un inodilatador que disminuye resistencias vasculares y el flujo sistémico; se indicó continuar con inotrópicos, manejo con ventilación mecánica (presión inspiratoria pico $21 \mathrm{~cm} / \mathrm{H} 2 \mathrm{O}$, presión positiva al final de la espiración 5, frecuencia respiratoria 50, fracción inspirada de oxígeno $100 \%$ ). El pecante tuvo sangrado de tubo digestivo (hematemesis y melena) y finalmente paro cardio-respiratorio sin respuesta a maniobras de reanimación.

\section{COMENTARIO CLÍNICO}

Para fines académicos dividimos el caso clínico en: manejo del recién nacido con diagnóstico de síndrome de Down y factores de riesgo de desarrollo de enterocolitis necrosante. Dentro del abordaje inicial de un paciente con características clínicas de síndrome de Down al nacimiento se deben solicitar: biometría hemática, pruebas de función tiroidea y valoración cardiológica. Existen otras valoraciones que deben realizarse con posterioridad, pero idealmente durante en los primeros tres meses de vida (audiología, oftalmología y genética). ${ }^{1}$

El motivo principal de traslado de este paciente fueron las alteraciones encontradas en la biometría hemática de nacimiento; en este caso, Ilamó la atención la trombocitopenia reportada en los tres estudios realizados. No se reportaron otros datos sugerentes de síndrome mieloproliferativo neonatal transitorio, las cuales se pueden esperar hasta en un $10 \%$ de los recién nacidos con esta condición; ${ }^{2}$ ante estos hallazgos en la biometría hemática se debió realizar aspirado de medula ósea, pero la gravedad del paciente no lo permitió.

El paciente tuvo restricción del crecimiento intrauterino con un peso al nacimiento de $1.820 \mathrm{~kg}$ talla de $43 \mathrm{~cm}$. El peso bajo para la edad gestacional se ha descrito ampliamente en las alteraciones cromosómicas. Se considera que las aneuploidias pueden tener un efecto sobre el ciclo celular en relación con su proliferación celular, y este defecto puede estar relacionado con las alteraciones del desarrollo y crecimiento. ${ }^{3} \mathrm{Se}$ deben utilizar graficas percentilares específicas para esta población, pero en Latinoamérica no existen parámetros poblacionales, por lo que se sugiere utilizar las gráficas españolas de peso y talla en población con síndrome de Down, ya que muestran mayor semejanza con nuestra población que las anglosajonas.

Independiente de los hallazgos cardiológicos encontrados a la exploración física al nacimiento, todo paciente con síndrome de Down requiere 
contar con un ecocardiograma, muchas cardiopatías pueden ser silentes en el periodo neonatal. En esta población se presentan en más del $50 \%$ de los casos, las cardiopatías más frecuentes en nuestro país son: comunicación inter-auricular, comunicación interventricular y persistencia del conducto arterioso. ${ }^{4}$

Tomando en cuenta los antecedentes de este paciente, pueden describirse algunos factores de riesgo que condicionaban el desarrollo de enterocolitis necrosante:

Cardiopatía congénita: el paciente tenía diagnóstico de tetralogía de Fallot, la cual se presenta en menos de $10 \%$ de las personas con síndrome de Down. Se ha demostrado que las cardiopatías congénitas se presentan en el $3 \%$ de los neonatos fallecidos por enterocolitis necrosante, la insuficiencia cardiaca se asocia a la producción de endotoxinas sistémicas, citocina miocárdica e incremento de TNF-alfa. Este aumento de factores de inflamación conduce a mayor susceptibilidad a enfermedades con componente inflamatorio entre las que se incluye la enterocolitis. ${ }^{5}$ Nuestro paciente ingreso con una PCR de $10.78 \mathrm{mg} / \mathrm{dL}$.

Peso bajo: en la enterocolitis necrosante la mucosa intestinal es dañada por las bacterias entéricas con posibilidades de producir afección de toda la pared con necrosis intestinal, septicemia y muerte, como en este caso. El riesgo de enterocolitis es inversamente proporcional a la edad gestacional y el peso bajo, los cuales son factores de riesgo que sugieren que la inmadurez de la mucosa intestinal es un factor predisponente importante. ${ }^{6}$

Inmadurez intestinal: hasta el $77 \%$ de los niños con síndrome de Down tienen asociadas anomalías gastrointestinales, que pueden ser de naturaleza estructural o funcional. Se ha demostrado que los procesos que afectan al sistema nervioso entérico no sólo afectan a la microanatomía, sino también a la función nerviosa, y hay algunas evidencias histológicas de las variaciones existentes con el resto de la población. Esto sugiere que los trastornos del desarrollo del sistema nervioso entérico son probablemente fundamentales para las alteraciones gastrointestinales funcionales. Los factores neurotrópicos que afectan al desarrollo del cerebro durante la embriogénesis probablemente están interrelacionados con el desarrollo del sistema nervioso entérico, pero aún no se ha establecido el mecanismo preciso de cómo ocurre esto. Las alteraciones motoras funcionales del esófago y del colon tienen alta prevalencia en esta población y pueden ser congénitas o adquiridas. Las más frecuentes incluyen síndromes de dismotilidad esofágica (acalasia, reflujo gastroesofágico, disfagia), así como una mayor incidencia de estreñimiento crónico y enfermedad de Hirschsprung (2-15\%). La mayoría de estos trastornos se asocia a inmadurez anatómica y funcional del sistema gastrointestinal que pudiera tener relación con el desarrollo de enterocolitis necrosante. $^{7}$

Otro punto a considerar es la enfermedad de Hirschsprung, la cual es 40 veces más frecuente en la población con síndrome de Down. Se ha descrito que en algunos casos, y más en neonatos, el cuadro que aparece en primer lugar es una enterocolitis, después de un estreñimiento que no llamó demasiado la atención. Esta enterocolitis se produce porque a medida que el intestino se dilata, aumenta la presión intraluminal que hace disminuir la perfusión sanguínea de la pared alterándose la mucosa y sus mecanismos de barrera y transporte. El estancamiento permite la proliferación bacteriana y la actuación de antígenos alimentarios. La situación puede evolucionar rápidamente a una sepsis y perforación intestinal. La enterocolitis es responsable de la existencia de un $30 \%$ de mortalidad en la enfermedad de Hirschsprung. ${ }^{8}$ 
En la mayoría de las series existe una predominancia de afectación del género masculino como en este caso. ${ }^{9}$

Se ha descrito que más del $70 \%$ de los pacientes recibió algún tipo de alimentación industrializada antes de desarrollar enterocolitis necrosante. El paciente estuvo por tres días con alimentación con fórmula para prematuro. La administración de regímenes estandarizados con leche materna o fórmula isoosmolar (alimentación enteral mínima) en forma intermitente, con volúmenes pequeños y con progresión lenta reduce la incidencia de enterocolitis necrosante. ${ }^{8}$

La policitemia constituye un factor de riesgo debido a que alteraría la circulación esplácnica y el paciente ingresó con hematocrito de $59 .{ }^{8}$

\section{COMENTARIO ANATOMOPATOLÓGICO}

Se realizó autopsia total a neonato con síndrome de Down; los hallazgos macroscópicos principales fueron: cardiopatía congénita compleja de tipo tetralogía de Fallot caracterizada por comunicación interventricular de $0.6 \times 0.6 \mathrm{~cm}$, cabalgamiento de la aorta, hipertrofia del ventrículo derecho y estenosis de la arteria pulmonar (Figuras 1-2), además de arco aórtico a la derecha, colateral aorto-pulmonar y comunicación interauricular de $0.7 \mathrm{~cm}$.

Como parte de las complicaciones el paciente tuvo enterocolitis necrosante con neumatosis intestinal, perforación intestinal, peritonitis aguda fibrinopurulenta y adherencias firmes de colon transverso con el diafragma (Figura 3).

Había marcada hipoplasia tímica (peso observado de 1.5 g vs. 10.0-14.0 g peso esperado) que histológicamente correspondieron a involución aguda de timo (Figura 4). Como hallazgo adicional interesante se identificó una fusión hepato-adrenal derecha (Figura 5).

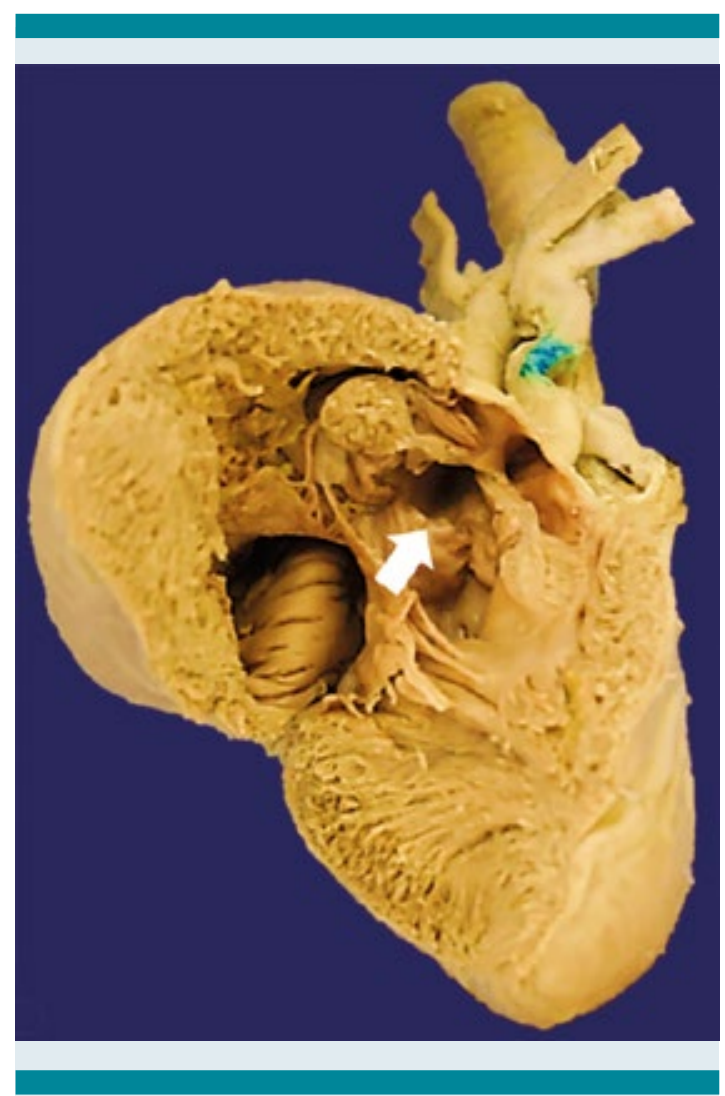

Figura 1. Aorta cabalgada en azul y estenosis de la arteria pulmonar (flecha).

\section{Diagnósticos anatómicos}

- Síndrome de Down

- Cardiopatía congénita compleja

- Enterocolitis necrosante con neumatosis intestinal

- Choque séptico y CID

- Hipoplasia tímica

- Depleción linfoide acentuada del timo, bazo y ganglios linfáticos

- Fusión hepático-suprarrenal derecha

- Citrobacter younge en la mayoría de los tejidos 


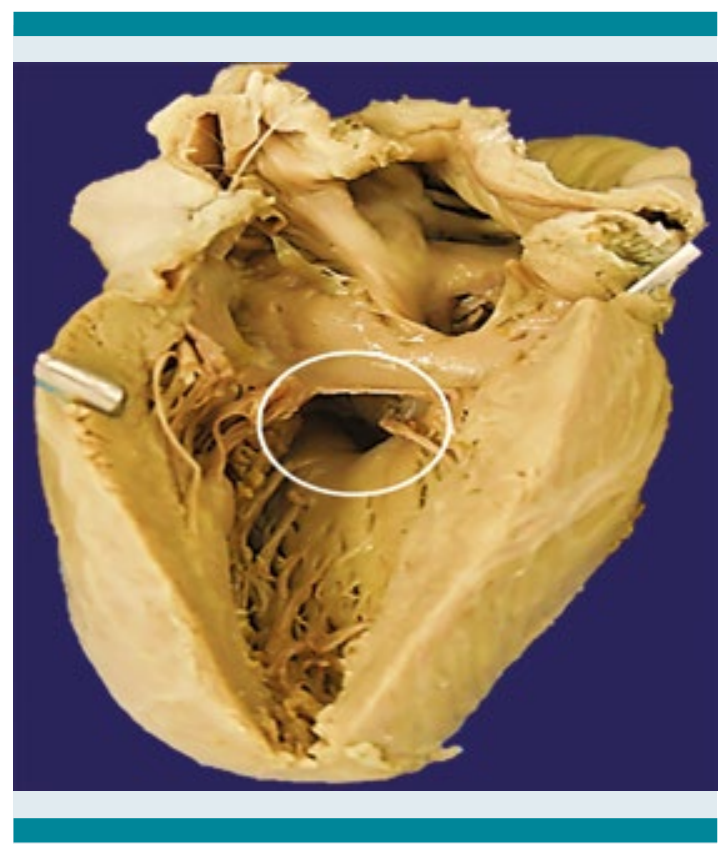

Figura 2. Comunicación interventricular de 0.6 cm.

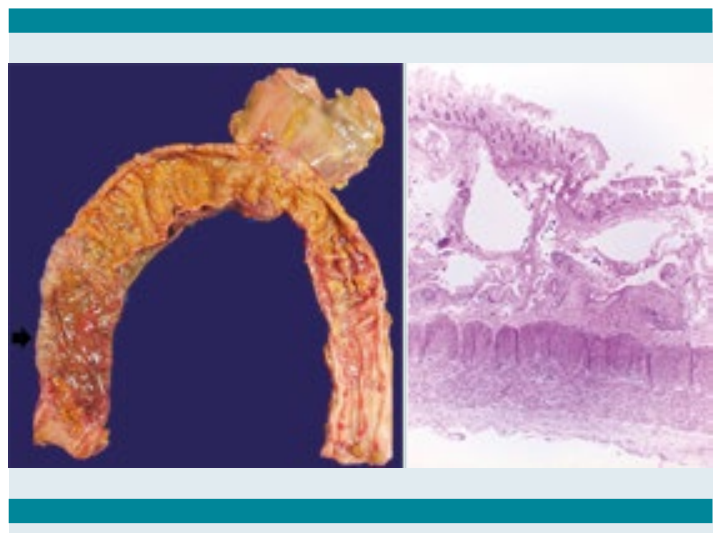

Figura 3. Colon con mucosa de aspecto en burbujas y serosa adherida al diafragma, histológicamente la mucosa tiene necrosis y hay neumatosis intestinal.

En nuestro paciente existió concordancia entre el abordaje clínico y tratamiento ofrecido; sin embargo, se debe tomar en cuenta que los pacientes con síndrome de Down presentan 30\% más riesgo de hospitalización por infecciones graves y mortalidad por septicemia que el resto de la población, ${ }^{10}$ lo cual puede estar relacionado con anomalías del sistema inmune.

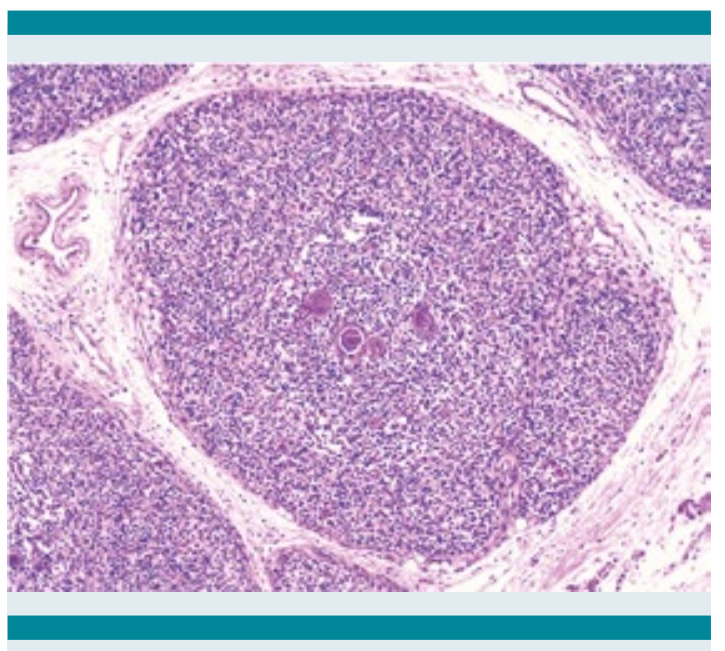

Figura 4. Timo con disminución del componente lifoide, incremento del estroma y corpúsculos de Hassal.

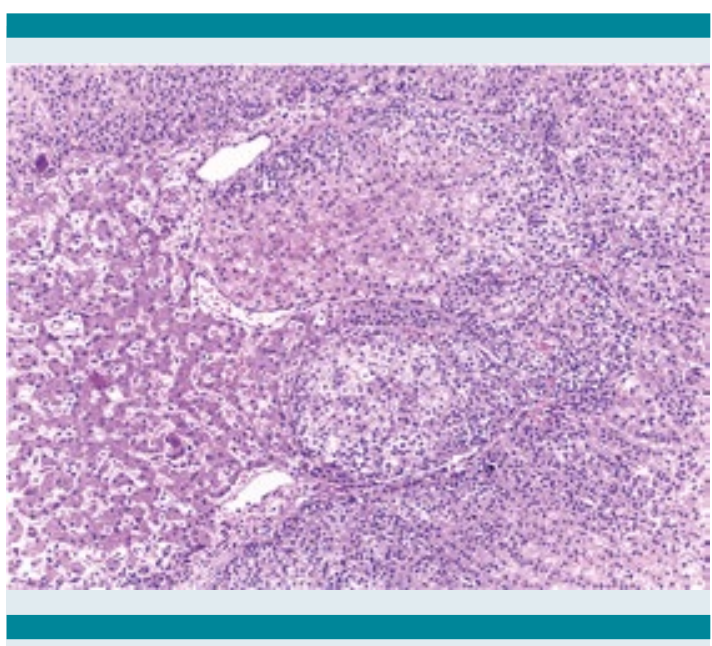

Figura 5. Fusión hepato-adrenal.

Dentro de los hallazgos anatomopatológicos se encontró hipoplasia tímica, las alteraciones inmunológicas de hipofunción tímica se encuentran desde etapas tempranas del desarrollo intrínseco del sistema inmune, ${ }^{11}$ lo que se debe tomar en cuenta ante un paciente con esta condición y mala evolución.

Se aisló postmortem en la mayoría de los tejidos Citrobacter younge, bacilo Gram negativo aero- 
bio, que se encuentra como flora saprófita en el tracto gastrointestinal, el cual se relaciona como causa frecuente de infecciones importantes, especialmente en sujetos inmunodeprimidos, ya que destruye las microvellosidades, formando lesiones de adherencia y eliminación.

A pesar de la experiencia creciente en el tratamiento multidisciplinario de esta patología, y siendo nuestro instituto un hospital de alta complejidad donde la mayoría de los neonatos que se derivan presentan patologías concurrentes, se deben tener especial atención en pacientes con síndrome de Down, ya que presentan mayor comorbilidad.

\section{REFERENCIAS}

1. MarilynJ. Bull, MD. Health Supervision for Children with Down Syndrome. Pediatr. 2011;128(2):393-406.

2. Mateos MK, Barbaric D, Byatt S-A, Sutton R, Marshall GM. Down syndrome and leukemia: insights into leukemogenesis and translational targets. Translational Pediatrics. 2015;4(2):76-92. doi:10.3978/j.issn.22244336.2015.03.03.
3. Nikkilä A, Källén B and Marsál K. Fetal growth and congenital malformations. Ultrasound Obstet Gynecol 2007;29:289-95.

4. Rubens J, Del Pozzo M.Calderón C. Malformaciones cardíacas en los niños con síndrome de Down Rev Esp Cardiol. 2003;56 (9):894-9.

5. Giannone PJ, Luce WA, Nankervis CA, et al. Necrotizing enterocolitis in neonates with congenital heart disease. Life Sci. 2008; 82: 341-347.

6. Niño DF, Sodhi $C P$, Hackam DJ. Necrotizing enterocolitis: new insights into pathogenesis and mechanisms. Nature reviews Gastroenterology \& hepatology. 2016;13(10):590600.

7. Moore SW. Down syndrome and the enteric nervous system. Pediatr Surg Int. 2008 Aug; 24(8):873-83. Epub 2008 Jul 17.

8. Lu P, Sodhi CP, Hackam DJ. Toll-like receptor regulation of intestinal development and inflammation in the pathogenesis of necrotizing enterocolitis. Pathophysiology. 2014; 21(1):81-93.

9. Neu J, Walker A. Necrotizing enterocolitis. N Engl J Med. 2011; 364: 255-264

10. Garrison, MM. Jeffries, H. Christakis, DA. Risk of death for children with Down syndrome and sepsis. J pediatr 2005;147:748-752.

11. Lima, et al. Decreased AIRE Expression and Global Thymic Hypofunction in Down Syndrome. The Journal of Immunology. 2011;187:2-8. 